\title{
Distal root curvatures in mandibular molars: analysis using digital panoramic X-rays
}

\author{
R. Fuentes 1,2 , C. Farfán ${ }^{3}$, N. Astete ${ }^{3}$, P. Navarro², A. Arias 1, 2, 4 \\ ${ }^{1}$ Department of Integral Adults Dentistry, Faculty of Dentistry, Universidad de La Frontera, Temuco, Chile \\ ${ }^{2}$ Research Centre for Dental Sciences (CICO), Faculty of Dentistry, Universidad de La Frontera, Temuco, Chile \\ ${ }^{3}$ Dental School, Faculty of Dentistry, Universidad de La Frontera, Chile \\ ${ }^{4}$ Universidad Adventista de Chile, Chillán, Chile
}

[Received: 28 March 2017; Accepted: 12 May 2017]

Background: The aim of this study was to describe the degree of curvature in distal roots in the first and second permanent mandibular molars in a Chilean patient sample. A cross-sectional descriptive study was conducted in which digital panoramic $X$-rays were analysed.

Materials and methods: Examinations of patients under 18 years, with signs of distortion or alteration in the contrast or the presence of pathologies that affected visualisation of the roots and pulp-chamber floor of the teeth to be analysed were excluded. Using the AutoCad software, an angle was drawn to represent the curve of the root in its different thirds, drawing lines inside the root canal from the pulp-chamber floor to the dental apex. Using the classic definition of dilaceration (root curvature $>90^{\circ}$ ), its prevalence was established. 412 teeth and roots were analysed, finding a dilaceration prevalence of $0.73 \%(n=3) .84 .72 \%$ of the roots presented some type of curvature.

Results: The middle third had the highest percentage of curvatures and the greatest average of angular curvature, whereas the cervical third was the straightest. No significant differences were found between the degree of curvature and the gender of the subjects, except for the apical third of tooth 3.6. The analysis of curvature by root third offers to the clinician a better perspective of the directional change of the roots and does not limit it to just the presence of curves in the apical third.

Conclusions: The report of the angular degree of the curvatures, in addition to the prevalence of dilacerations, informs to the clinicians about the likelihood of finding difficulties when treating root canals. (Folia Morphol 2018; 77, 1: 131-137)

Key words: root curvatures, dilacerations, digital panoramic X-rays, morphometry

\section{INTRODUCTION}

Detailed knowledge of the dental anatomy, particularly the root, is relevant for some areas of dentistry. This is the case of endodontics, where the access, preparation and identification of the root canals pose a great challenge to the specialist [12].
Estimating the degree of root curvatures makes it possible to establish normality parameters and thus identify cases of excessively pronounced curvatures [6], because it is known that each permanent tooth has a wide range of variations in root morphology [26]. These excessive curvatures, or dilacerations, are the 
result of a disturbance in the root formation that produces a deviation in the linear relation between the crown of a tooth and its root [6]. The causes of root dilacerations vary depending of tooth type and dentition. In permanent anterior teeth, dilacerations most often is a consequence of a trauma to the primary predecessor, such as an intrusive trauma, and produce a dislocation of the radicular parts of the germ [16, 24]. On the other hand, the causes of root dilacerations of premolars and molar is not completely clear, although it is often seen when the roots of retained teeth encounter a cortical bone structure during its development [16].

Hamasha et al. [10] and Malcic et al. [17] define "dilaceration" as a deviation of the root in a mesial or distal direction, forming an angle of $90^{\circ}$ or more with the normal longitudinal axis of the tooth. Chohayeb [5], by contrast, define dilaceration as an apical deviation equal to or greater than $20^{\circ}$ in relation to the central axis of the tooth. There have been few descriptions of the degree of curvatures and the prevalence of dilacerations in the roots of permanent teeth [10], and these vary according to the criteria used to classify them [9]. Çolak et al. [6] report a prevalence of dilacerations of $0.78 \%$ in a sample of 192,150 teeth, among which they emphasize the third and second mandibular molar with the highest percentages $(3.76 \%$ and $1.81 \%$, respectively). On the other hand, Hamasha et al. [10] report a prevalence of dilacerations of $3.78 \%$ in a sample of 4,655 teeth, where the third and first mandibular molar showed the highest prevalences $(19.2 \%$ and $5.6 \%$, respectively), followed by the second maxillary premolar and second mandibular molar $(4.7 \%$ and $3.6 \%$, respectively). Nabavizadeh et al. [20] report a prevalence of dilacerations of $0.3 \%$ in a sample of 6,146 teeth, where the second and first mandibular molar presented the highest frequencies $(1.6 \%$ and $0.7 \%$, respectively).

An X-ray is required to diagnose a dilaceration correctly [10], and the periapical X-ray is considered to be the most suitable method [20]. X-rays represent a bidimensional image of a three-dimensional (3D) structure, which is why it is necessary to consider that some morphological characteristics will not be accurately reflected. This is an obstacle in the diagnosis of curvatures that occur on the vestibular-palatal or vestibular-lingual axis, and even when analysing roots superimposed on the image [7]. Cone beam computed tomography (CBCT) has solved this limitation and more accurate studies have been conducted [11]; despite its advantages, however, this technology is not always available in the clinic. In contrast to $C B C T$, the panoramic $\mathrm{X}$-ray is a highly requested examination and commonly used by the dentist. The panoramic $\mathrm{X}$-ray in its digital format has some advantages over other methods to study root curvatures, because it requires low doses of radiation, it allows a simultaneous assessment of several structures, and it is economical [9]. For these reasons, the aim of this study is to determine the degree of curvature of each third of the distal roots of the first and second permanent mandibular molar, analysed using digital panoramic $X$-rays in a Chilean patient sample.

\section{MATERIALS AND METHODS}

A cross-sectional descriptive study was conducted, in which a database of digital panoramic X-rays (1:1 ratio) available in the Imaging Unit of the Teaching Dental Clinic in the Faculty of Dentistry, Universidad de la Frontera, Temuco, Chile was analysed. The $\mathrm{X}$-rays were taken using a standard technique with the PAX-400C orthopantomograph (VATECH, Korea, 2010) in 2014. This study has the approval of the Scientific Ethics Committee of the Universidad de La Frontera (file $\mathrm{N}^{\circ} 015 / 2014$ ).

The gender and age of the individuals available in the examination file was recorded. All the X-rays from subjects under 18 years, $X$-rays with signs of distortion or alteration in the contrast, signs compatible with pathologies that affect visualisation by the root (cysts, internal root resorption, external root resorption and fractures), presence of teeth included in the molar zone in a severely bad position or with restorations that compromise the visibility of any root third including the pulp-chamber floor (root canals, posts, orthodontic bands, etc.), presence of titanium plates or evidence of orthognathic surgery were excluded. 140 subjects (91 women; 49 men) whose age average was $32.74 \pm 13.94$ years (18-69 years) were included in this study. 412 teeth were examined in total, of which 200 were first mandibular molars (100 teeth from the right hemiarch and 100 teeth from the left hemiarch), and 212 were second mandibular molars (106 from the right hemiarch and 106 from the left hemiarch).

All the digital panoramic X-rays were projected onto a 29" LED monitor (LG, model 29UM67) with a resolution of $2560 \times 1080$ pixels, and analysed by two examiners. The degree of curvature of the distal 


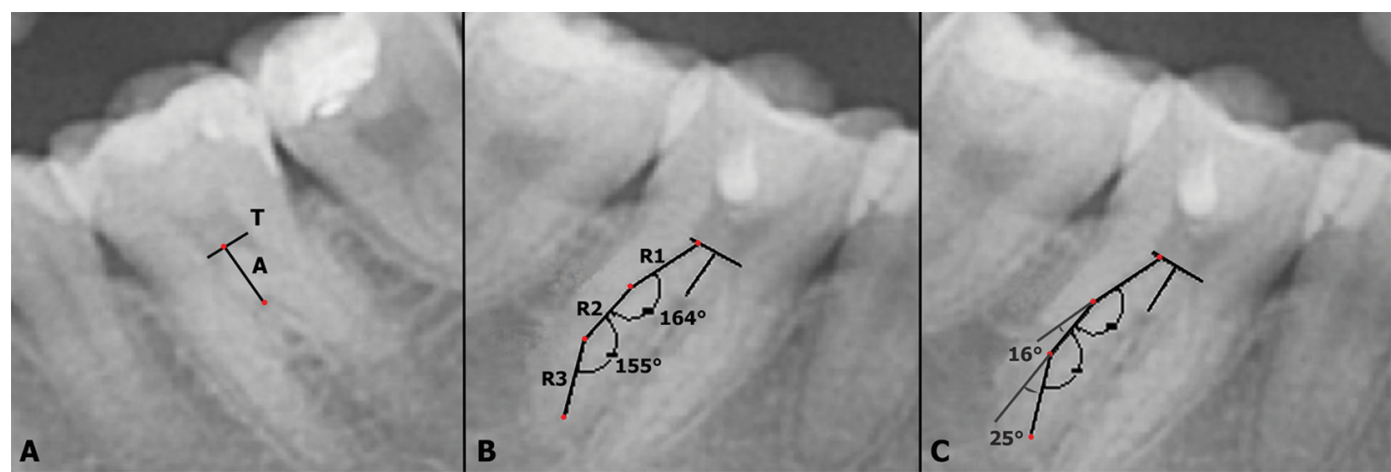

Figure 1. Methods for draw the angle that represents the curve of the root for each thirds. A. Two points were drawn in the furcation of the tooth and on the chamber floor to determine the longitudinal axis of the tooth (A). Then, a tangent line (T) to the chamber floor was drawn in a way that would be perpendicular to the axis of the tooth; B. A line (R1) was drawn from the midpoint of the distal root canal on the same plane as line T. R1 is a straight line that follow the direction of the root, in the centre of the canal, to the point at which the line left the canal. A second line (R2) was drawn in the same way as the previous one, until finally reach the root apex; $\mathbf{C}$. The angle considered for the registry of the curvature was the additional or inferior angle.

roots visible on the panoramic X-ray was determined for the first and second mandibular molar of each hemiarch. Analysis of the mesial root of these teeth was excluded due to the superimposition of structures on the image. To take the measurements, the AutoCAD software was used (2010 version; Autodesk Inc. USA), which processes vector images and can incorporate photographic or bitmap files, making it possible to draw basic figures, lines, arcs and rectangles, to measure distances and angles and to calculate other geometric parameters. The size of the X-ray was adjusted by the measurement software as per the parameters indicated by the orthopantomograph software so that the measurement scale was not altered. Using the software's drawing tool, an angle was drawn that represents the curve of the root for each of its thirds through two lines. The lines were drawn as follows:

- the longitudinal axis of the tooth $(A)$ was determined through the projection of two points: one located in the furcation of the tooth (most concave point) and the other located on the chamber floor above the furcation and at the midpoint of the mesiodistal distance from the chamber (Fig. 1A);

- a line tangent $(T)$ to the chamber floor was drawn in a way that would be perpendicular to the axis of the tooth (Fig. 1A);

- the lines of the angle were drawn to measure, establishing a midpoint in the distal root canal on the same plane as line $\mathrm{T}$;

- a line (R1) was drawn from this point following the direction of the root, in the centre of the canal, to the point at which the line left the canal (Fig. 1B);
- from the point at which R1 left the canal, a second line (R2) was drawn, following the direction of the root, in the centre of the canal, to the point at which the line again left the canal (Fig. 1B).

This procedure was repeated until the necessary angles were drawn that represent the curvatures of a root in its different thirds, until finally reaching the root apex (Fig. 1B). The angle considered for the registry of the curvatures was the additional one to that formed by both lines or inferior angle (Fig. 1C). Those thirds in which no curvatures were found were described with an angle equal to $0^{\circ}$ and the criterion of Hamasha et al. [10], Malcic et al. [17] and Colak et al. [6] was used to identify dilacerations (degree of curvature greater than or equal to $90^{\circ}$ ); therefore, all those root thirds that presented an angle greater than $0^{\circ}$ and less than $90^{\circ}$ were considered curved.

\section{Statistical analysis}

In order to determine inter-operator agreement, the intraclass correlation coefficient (ICC) was calculated to measure the curvatures and a kappa statistic was used to identify curvatures according to root third by means of the individual analysis of 50 dental roots of first and second molars by each operator. Mean and standard deviation were used for the data analysis. The t-test for independent samples was performed with a significance level of 0.05. The data were analysed using the SPSS 20.0 software. Associations were sought between the degree of curvature of the roots in their different thirds and the variables gender and dental group. 
Table 1. Average of angular curvature of each root third (cervical, middle and apical) by tooth, finding of dilacerations, presence of straight root thirds, and the count and its respective percentage

\begin{tabular}{|c|c|c|c|c|c|c|c|c|c|c|}
\hline \multirow{2}{*}{\multicolumn{2}{|c|}{ Tooth }} & \multicolumn{3}{|c|}{ Cervical } & \multicolumn{3}{|c|}{ Middle } & \multicolumn{3}{|c|}{ Apical } \\
\hline & & Straight & Curvature & Dilaceration & Straight & Curvature & Dilaceration & Straight & Curvature & Dilaceration \\
\hline & $\%$ & $97 \%$ & $3 \%$ & - & $33 \%$ & $67 \%$ & - & $65 \%$ & $34 \%$ & $1 \%$ \\
\hline \multirow[t]{3}{*}{3.6} & $\mathrm{~N}$ & 97 & 3 & - & 33 & 67 & - & 65 & 34 & 1 \\
\hline & Angle & $0^{\circ}$ & $1.01^{\circ}$ & - & $0^{\circ}$ & $11.90^{\circ}$ & - & $0^{\circ}$ & $9.77^{\circ}$ & $114^{\circ}$ \\
\hline & $\%$ & $93.4 \%$ & $5.7 \%$ & $0.9 \%$ & $46.2 \%$ & $53.8 \%$ & - & $59.5 \%$ & $39.6 \%$ & $0.9 \%$ \\
\hline \multirow[t]{3}{*}{3.7} & $\mathrm{~N}$ & 99 & 6 & 1 & 49 & 57 & - & 63 & 42 & 1 \\
\hline & Angle & $0^{\circ}$ & $1.07^{\circ}$ & $162^{\circ}$ & $0^{\circ}$ & $10.08^{\circ}$ & - & $0^{\circ}$ & $10.92^{\circ}$ & $135^{\circ}$ \\
\hline & $\%$ & $91 \%$ & $9 \%$ & - & $28 \%$ & $72 \%$ & - & $63 \%$ & $37 \%$ & - \\
\hline \multirow[t]{3}{*}{4.6} & $\mathrm{~N}$ & 91 & 9 & - & 28 & 72 & - & 63 & 37 & - \\
\hline & Angle & $0^{\circ}$ & $1.62^{\circ}$ & - & $0^{\circ}$ & $13.93^{\circ}$ & - & $0^{\circ}$ & $8.14^{\circ}$ & - \\
\hline & $\%$ & $92.5 \%$ & $7.5 \%$ & - & $33 \%$ & $67 \%$ & - & $66 \%$ & $34 \%$ & - \\
\hline \multirow[t]{2}{*}{4.7} & $\mathrm{~N}$ & 98 & 8 & - & 35 & 71 & - & 70 & 36 & - \\
\hline & Angle & $0^{\circ}$ & $1.88^{\circ}$ & - & $0^{\circ}$ & $13.55^{\circ}$ & - & $0^{\circ}$ & $8.46^{\circ}$ & - \\
\hline
\end{tabular}

Table 2. Average of angular curvature of homologous teeth grouped according to root third (cervical, middle and apical).

\begin{tabular}{lccc}
\hline Homologous teeth & \multicolumn{3}{c}{ Average of angular curvature } \\
\cline { 2 - 4 } & Cervical & Middle & Apical \\
\hline $3.6+4.6(\mathrm{n}=200)$ & $1.32^{\circ}$ & $12.92^{\circ}$ & $8.96^{\circ}$ \\
$3.7+4.7(\mathrm{n}=212)$ & $1.48^{\circ}$ & $11.82^{\circ}$ & $9.69^{\circ}$ \\
\hline
\end{tabular}

\section{RESULTS}

In the analysis of inter-operator agreement, the ICC was $96.9 \%$ (very good agreement), while the kappa statistic was $100 \%$ (very good agreement).

The prevalence of dilacerations found was $0.73 \%$ $(n=3)$ and the percentage of roots that presented at least one curvature was $84.72 \%(n=347)$. The average angle of the curvatures of each tooth according to the root third in which they were found is summarised in Table 1. For all the first and second mandibular molars analysed (teeth 3.6, 3.7, 4.6 and 4.7), the middle third was the one with the highest percentage of curvatures $(67 \%, 53.8 \%, 72 \%$ and $67 \%$, respectively), whereas the cervical third was the straightest $(97 \%, 93.4 \%, 91 \%$ and $92.5 \%$, respectively). The middle third of teeth 4.6 and 4.7 had the highest average of angular curvature $\left(13.93^{\circ}\right.$ and $13.55^{\circ}$, respectively).

Only three cases of teeth with root thirds with degrees of curvature greater than $90^{\circ}$ (dilacerations) were found: one in the cervical third of a 3.7 tooth $\left(162^{\circ}\right)$ and two in the apical third of a 3.6 and a 3.7 tooth $\left(114^{\circ}\right.$ and $135^{\circ}$, respectively). The average angle of the curvatures of each third for the teeth grouped by type is shown in Table 2 . The average angle of curvatures of first mandibular molars (3.6 and 4.6) in their cervical, middle and apical root thirds was $1.32^{\circ}, 12.92^{\circ}$ and $8.96^{\circ}$, respectively. Also, the average angle of curvatures of the second mandibular molars (3.7 and 4.7 ) was $1.48^{\circ}, 11.82^{\circ}$ and $9.69^{\circ}$ for their root thirds, respectively.

Table 3 presents the average angle of curvatures for each third of the teeth analysed, grouped according to gender. No significant differences were found for the degrees of curvature of the different root thirds between men and women, except for the apical third of tooth $3.6(p=0.023)$ that was greater in men.

\section{DISCUSSION}

In this study a novel analysis was made of the curvatures present in the different thirds of the distal roots of the first and second mandibular molars seen 
Table 3. Average of angular curvature of each root third by tooth and according to gender

\begin{tabular}{|c|c|c|c|c|c|c|c|}
\hline \multirow[t]{2}{*}{ Tooth } & \multirow[t]{2}{*}{ Gender } & \multicolumn{6}{|c|}{ Root third } \\
\hline & & Cervical & $\mathbf{p}$ & Middle & $\mathbf{p}$ & Apical & p \\
\hline \multirow{2}{*}{3.6} & Male & $2.21^{\circ}$ & \multirow{2}{*}{0.285} & $11.59^{\circ}$ & \multirow{2}{*}{0.824} & $14.97^{\circ}$ & \multirow{2}{*}{$0.023^{*}$} \\
\hline & Female & $0.39^{\circ}$ & & $12.06^{\circ}$ & & $7.17^{\circ}$ & \\
\hline \multirow{2}{*}{3.7} & Male & $1.32^{\circ}$ & \multirow{2}{*}{0.562} & $10.49^{\circ}$ & \multirow{2}{*}{0.782} & $13.28^{\circ}$ & \multirow{2}{*}{0.088} \\
\hline & Female & $0.93^{\circ}$ & & $9.87^{\circ}$ & & $9.70^{\circ}$ & \\
\hline \multirow{2}{*}{4.6} & Male & $2.14^{\circ}$ & \multirow{2}{*}{0.492} & $13.86^{\circ}$ & \multirow{2}{*}{0.960} & $7.83^{\circ}$ & \multirow{2}{*}{0.853} \\
\hline & Female & $1.34^{\circ}$ & & $13.97^{\circ}$ & & $8.31^{\circ}$ & \\
\hline \multirow{2}{*}{4.7} & Male & $1.49^{\circ}$ & \multirow{2}{*}{0.646} & $12.80^{\circ}$ & \multirow{2}{*}{0.612} & $5.68^{\circ}$ & \multirow{2}{*}{0.065} \\
\hline & Female & $2.12^{\circ}$ & & $14.02^{\circ}$ & & $10.22^{\circ}$ & \\
\hline
\end{tabular}

${ }^{*}$ Statistically significant differences

through digital panoramic X-rays. Analysis of the curvatures by root third offers a better approach to study the root anatomy through images, because it provides a perspective of the directional change of the roots, which must be identified by the clinician prior to treating the tooth. To our best knowledge there are no other studies that analyse the root curvatures this way using panoramic X-rays.

The root dilacerations can occur by multiple and sometimes unknown causes, like a trauma in the predecessor tooth or obstacles in the development of the tooth root sheath. Another hypothesis that could explain this alteration is relative to bone remodelling gradients in alveolar bone (gradients of plasticity) [23], which could be the cause of a displacement of tooth root. The role of tooth root sheath or Hertwig's epithelial root sheath in root formation is well known and accepted [27] and its interaction with bone plasticity zones, characterised by a great amount of localised remodelling, has been hypothesised [23]. According to this hypothesis, during the physiologic growth related to alveolar and basal bones, portions of developing tooth could be found within one or more of these plasticity zones and a root dilacerations may result by a tooth movement between two zones with different plasticity rates [23].

The prevalence of dilacerations reported in the literature varies according to the type of population studied (origin), type and number of teeth analysed, method of diagnosis and the specific definition of dilaceration. Miloglu et al. [18] report a prevalence of dilacerations $\left(>90^{\circ}\right)$ of $4.3 \%(n=276)$ in a Turkish sample population $(6,386$ permanent teeth of all types) using periapical X-rays, whereas Bodrumlu et al. [3] report a prevalence of $3.4 \%(n=317)$ in the same population sample $(9,406$ permanent teeth of all types) using the same method. Also, Udoye and Jafarzadeh [25] report a prevalence of dilaceration of $2.97 \%\left(>90^{\circ}\right)$ in a Nigerian sample population (706 permanent teeth of all types) using periapical X-rays. In our study, the prevalence of dilacerations found was smaller than the previous studies reaching $0.73 \%(n=3)$ in a sample of 412 teeth (first and second mandibular molars), where only the distal roots were analysed.

On the other hand, Aminsobhani et al. [1] use a 3D method (CBCT) to identify curvatures (among other anatomical findings) in anterior teeth, emphasizing its high precision over other methods for studying roots. In this same line, Estrela et al. [8] propose a method to determine the root curvature using $C B C T$ and basing it on the calculation of the radius of a circumference, to then report in 2015 [7] the prevalence of curvatures in the cervical and apical thirds of the mesial and distal roots of molars in a Brazilian sample. That study, like ours, proposes a new approach for analysing curvatures, considering that these not only originate in the apical third, but can also occur in the upper thirds. Their study reports that $92.75 \%$ of the analysed roots present curvature in the apical third, and that $73.25 \%$ show it in the cervical third (using the coronal plane of the CBCT as a reference), which is compatible with our findings (84.72\% of the analysed teeth presented at least one curvature in one of its thirds). Llena et al. [15], in a Spanish sample and using $\mathrm{CBCT}$, report that $65 \%$ 
of the premolars analysed possess some degree of curvature, which reaffirms the need to know not only the cases of dilaceration, but also those cases in which curvatures exist that could require greater attention on the part of the clinician. There is no doubt that a 3D method for analysis of root curvatures is most adequate to understand its complex anatomy, which is compound by several flexion in three different planes; however its application is very limited by economical and technician factors. Our study proposes a more accessible method for this purposes, using digital panoramic X-rays as a tool; however, its use is limited and can be offers some inaccuracies when the tooth has curvatures in a vestibular or lingual/palatine direction. This is an important method limitation in the use of bidimensional images, but the justification of its use is based on the high prevalence of dilacerations and curvatures in mesial or distal direction.

Finally, the criterion used to differentiate a curvature from a dilaceration of the root has varied over the years and this may in part explain the wide range of reported percentages [16]. Schneider [21] defines a way to measure the angle of deviation of the root with respect to the longitudinal axis of the tooth, thereby classifying the roots as straight, moderately or severely curved. That method has been replicated constantly in the literature and there has been a succession of classifications. Chohayeb [5] define a dilaceration as a deviation of $20^{\circ}$ or more of the apical portion of the root during an analysis of the morphology of the superior lateral incisors. Later, a consensus was established to define a dilaceration as a deviation of the root in a mesial or distal direction, forming an angle of $90^{\circ}$ or more with respect to the longitudinal axis of the tooth or root $[6,10,17]$.

Generally, the reports agree in that there are no statistical differences in the dilaceration of dental roots according to the age of the subjects when permanent teeth are studied [3]. This is because once root development is complete, from 15 years of age no further dental morphological changes occur and hence in this study that analysis has been disregarded. When examining the degree of root curvature in molars of men and women, we did not find any significant differences, which is consistent with other reports that analyse different dental groups $[3,10$, $15,18]$. On this last point, it is possible to find some studies that do indeed report significant differences in the prevalence of root dilacerations between men and women [6], although the degree of the curvatures of the teeth has not been explored and they are not classified as dilacerated.

A large body of evidence regarding the root morphology is comprised of case reports $[2,4,13,14$, $19,22]$ and some prevalence studies $[3,6,10,17]$. Our study not only reports the prevalence found of root dilacerations, but also provides a novel report on the degree of angular curvature and the directional change that occurs in the distal roots of mandibular molars. In a previous study, we reported on the angular degree of root curvature in mandibular premolars and what it means for the clinician to know both the directional change of the root, the magnitude and percentage of teeth or cases he/she may be facing [9].

\section{CONCLUSIONS}

Of all total distal roots of first and second mandibular molars analysed, there was a prevalence of dilacerations of $0.73 \%$ ( $>$ to $90^{\circ}$ ), whereas $84.72 \%$ of these roots presented some type of curvature in some of their thirds. The middle third had the highest percentage of curvatures (from $53.8 \%$ to $67 \%$ of the curves found for each type of tooth) and the highest average of angular curvature $\left(12.92^{\circ}\right.$ and $11.82^{\circ}$ for first and second mandibular molar, respectively), while the cervical third was the straightest.

It is important to consider that the curvatures, whether they are within a normal range or classified as a dilaceration, represent a directional change of the structure that the clinician must be able to identify in order to treat the tooth. These curvatures do not originate only at apical level and it is very common to find them at the level of the middle and even cervical third. Knowledge of the percentage of curved and straight roots in a certain dental group helps the clinician in decision-making and promotes caution when treating the tooth.

\section{Funding}

Financial support (partially) by the Office of Research, Universidad de La Frontera.

\section{REFERENCES}

1. Aminsobhani M, Sadegh M, Meraji N, et al. Evaluation of the root and canal morphology of mandibular permanent anterior teeth in an Iranian population by cone-beam computed tomography. J Dent (Tehran). 2013; 10(4): 358-366, indexed in Pubmed: 24396355.

2. Bhatia SK, Goyal A, Gauba K, et al. Unusual root development following surgical repositioning of horizontally developing central incisor. Dent Traumatol. 2015; 31(5): 
413-417, doi: 10.1111/edt.12182, indexed in Pubmed: 26040300.

3. Bodrumlu E, Gunduz K, Avsever $\mathrm{H}$, et al. A retrospective study of the prevalence and characteristics of root dilaceration in a sample of the Turkish population. Oral Radiol. 2012; 29(1): 27-32, doi: 10.1007/s11282-012-0105-1.

4. Bolhari B, Pirmoazen S, Taftian E, et al. A case report of dilacerated crown of a permanent mandibular central incisor. J Dent (Tehran). 2016; 13(6): 448-452, indexed in Pubmed: 28243307.

5. Chohayeb AA. Dilaceration of permanent upper lateral incisors: frequency, direction, and endodontic treatment implications. Oral Surg Oral Med Oral Pathol. 1983; 55(5): 519-520, indexed in Pubmed: 6575342.

6. Colak H, Bayraktar Y, Hamidi MM, et al. Prevalence of root dilacerations in Central Anatolian Turkish dental patients. West Indian Med J. 2012; 61(6): 635-639, indexed in Pubmed: 23441361

7. Estrela C, Bueno MR, Barletta FB, et al. Identification of apical and cervical curvature radius of human molars. Braz Dent J. 2015; 26(4): 351-356, doi: 10.1590/010364402013x0252, indexed in Pubmed: 26312971.

8. Estrela C, Bueno MR, Sousa-Neto MD, et al. Method for determination of root curvature radius using cone-beam computed tomography images. Braz Dent J. 2008; 19(2): 114-118, doi: 10.1590/s0103-64402008000200005, indexed in Pubmed: 18568224.

9. Fuentes $R$, Arias $A$, Navarro $P$, et al. Morfometría de Premolares Mandibulares en Radiografías Panorámicas Digitales; Análisis de Curvaturas Radiculares. Int J Morphol. 2015; 33(2): 476-482, doi: 10.4067/s071795022015000200012.

10. Hamasha AA, Al-Khateeb T, Darwazeh A. Prevalence of dilaceration in Jordanian adults. Int Endod J. 2002; 35(11): 910-912, indexed in Pubmed: 12453019.

11. Hong $X$, Zhang $\mathrm{Ru}$, Pan $\mathrm{H}$, et al. Lingual radicular rift valley in a mandibular right first premolar root: report of a rare case and review of the literature. Int J Morphol. 2013; 31(1): 338-344, doi: 10.4067/s071795022013000100052.

12. Jafarzadeh $\mathrm{H}$, Abbott PV. Dilaceration: review of an endodontic challenge. J Endod. 2007; 33(9): 1025-1030, doi: 10.1016/j. joen.2007.04.013, indexed in Pubmed: 17931926.

13. Kearns HP. Dilacerated incisors and congenitally displaced incisors: three case reports. Dent Update. 1998; 25(8): 339-342, indexed in Pubmed: 10478004.

14. Lin L, Dowden WE, Langeland K. Bilateral dilaceration. J Endod. 1982; 8(2): 85-87, doi: 10.1016/S00992399(82)80264-1, indexed in Pubmed: 6950021.

15. Llena C, Fernandez J, Ortolani PS, et al. Cone-beam computed tomography analysis of root and canal morphol- ogy of mandibular premolars in a Spanish population. Imaging Sci Dent. 2014; 44(3): 221-227, doi: 10.5624/ isd.2014.44.3.221, indexed in Pubmed: 25279343.

16. Luder HU. Malformations of the tooth root in humans. Front Physiol. 2015; 6: 307, doi: 10.3389/ fphys.2015.00307, indexed in Pubmed: 26578979.

17. Malcić A, Jukić S, Brzović V, et al. Prevalence of root dilaceration in adult dental patients in Croatia. Oral Surg Oral Med Oral Pathol Oral Radiol Endod. 2006; 102(1): 104-109, doi: 10.1016/j.tripleo.2005.08.021, indexed in Pubmed: 16831681.

18. Miloglu O, Cakici F, Caglayan F, et al. The prevalence of root dilacerations in a Turkish population. Med Oral Patol Oral Cir Bucal. 2010; 15(3): e441-e444, indexed in Pubmed: 20038903.

19. Monea M, Moldovan C. Rare Root Morphology of a Maxillary Central Incisor Associated With Gingival Hyperplasia: An Endodontic Case Report. Medicine (Baltimore). 2016; 95(18): e3617, doi: 10.1097/MD.0000000000003617, indexed in Pubmed: 27149498.

20. Nabavizadeh Mr, Sedigh Shamsi M, Moazami F, et al. Prevalence of root dilaceration in adult patients referred to shiraz dental school (2005-2010). J Dent (Shiraz). 2013; 14(4): 160-164, indexed in Pubmed: 24724139.

21. Schneider SW. A comparison of canal preparations in straight and curved root canals. Oral Surg Oral Med Oral Pathol. 1971; 32(2): 271-275, indexed in Pubmed: 5284110.

22. Smith DM, Winter GB. Root dilaceration of maxillary incisors. Br Dent J. 1981; 150(5): 125-127, indexed in Pubmed: 6937200.

23. Standerwick R. A possible etiology for the dilaceration and flexion of permanent tooth roots relative to bone remodeling gradients in alveolar bone. Dental Hypotheses. 2014; 5(1): 7-10, doi: 10.4103/2155-8213.128105.

24. Topouzelis N, Tsaousoglou P, Pisoka V, et al. Dilaceration of maxillary central incisor: a literature review. Dent Traumatol. 2010; 26(5): 427-433, doi: 10.1111/j.16009657.2010.00915.x, indexed in Pubmed: 20831640.

25. Udoye $\mathrm{Cl}$, Jafarzadeh $\mathrm{H}$. Dilaceration among Nigerians: prevalence, distribution, and its relationship with trauma. Dent Traumatol. 2009; 25(4): 439-441, doi: 10.1111/j.1600-9657.2009.00796.x, indexed in Pubmed: 19614739.

26. Vertucci FJ. Root canal anatomy of the human permanent teeth. Oral Surg Oral Med Oral Pathol. 1984; 58(5): 589-599, indexed in Pubmed: 6595621.

27. Zeichner-David M, Oishi K, Su Z, et al. Role of Hertwig's epithelial root sheath cells in tooth root development. Dev Dyn. 2003; 228(4): 651-663, doi: 10.1002/dvdy.10404, indexed in Pubmed: 14648842. 